\title{
Re-irradiation Using Intensity-modulated Radiotherapy for Recurrent and Second Primary Head and Neck Cancer
}

\author{
SEO HEE CHOI ${ }^{1}$, JEE SUK CHANG ${ }^{1}$, JINHYUN CHOI $^{1}$, SO HYUN PARK $^{1}$, \\ KI CHANG KEUM ${ }^{1}$, KYUNG RAN PARK $^{2}$ and CHANG GEOL LEE ${ }^{1}$ \\ ${ }^{1}$ Department of Radiation Oncology, Yonsei University College of Medicine, Seoul, Republic of Korea; \\ ${ }^{2}$ Department of Radiation Oncology, University of Washington Medical Center, Seattle, WA, U.S.A.
}

\begin{abstract}
Background/Aim: Information on re-irradiation (re-RT) for recurrent and second primary head and neck cancer is limited. Herein, a description of our long-term experience of re-RT for previously irradiated head and neck cancer is provided. Materials and Methods: A retrospective review was performed for 73 consecutive patients reirradiated for head and neck cancer between 2006 and 2015. $R e-R T$ targets encompassed only the recurrent gross tumor and had tight margins $(5-10 \mathrm{~mm})$. Results: Salvage surgery was performed on 28 (38\%) patients before re-RT and 53 (73\%) patients received chemotherapy concurrent with re-RT. The median interval between initial and re-RT was 31 months and the median cumulative dose of the two irradiations was 126 Gy (biologically equivalent to 2 Gy fractionation). With a median survival of 33 months, locoregional recurrence after re-RT developed in 37 patients $(51 \%$; 25 infield, 12 outfield). In multivariate analysis, higher re-RT dose ( $\geq 66 \mathrm{~Gy})$, longer time interval ( $>2$ years), and use of concurrent chemotherapy were associated with improved locoregional recurrence-free survival (all $p<0.05)$. Additionally, performance status, additional surgical resection, and longer interval were associated with better overall survival ( $p=0.006,0.021,0.004$, respectively). Clinically significant acute and late toxicities occurred in $14 \%$ and $22 \%$ of evaluable patients, but no grade 5 toxicity was observed. Conclusion: Moderate safety and acceptable toxicity was found after re-RT using tight margins, sufficient radiation dose, and daily image guidance. Encouraging local control and survival were obtained, similar to historical data using 1-2 cm margins.
\end{abstract}

Correspondence to: Chang Geol Lee, MD, Ph.D., Department of Radiation Oncology, Yonsei University College of Medicine, 50 Yonsei-ro, Seodaemun-gu, Seoul 120-752, Republic of Korea. Tel: +82 222288095, Fax: +82 23129033, e-mail: cglee1023@yuhs.ac

Key Words: Head and neck cancer, radiotherapy, re-irradiation, salvage therapy.
Currently, a multidisciplinary approach of surgery, radiotherapy (RT), and chemotherapy rather than single-modality therapy is used to manage locally advanced head and neck cancer. RT is a vital and effective complementary locoregional treatment modality. Postoperative RT with or without chemotherapy has been shown to enhance local control and improve survival for patients who underwent surgical resection $(1,2)$. Upfront RT with concomitant chemotherapy, which was originally pioneered for inoperable head and neck cancer, has emerged as a curative treatment option comparable to upfront surgery in operable patients $(3,4)$.

However, for patients with recurrent and second primary head and neck cancer occurring within areas previously irradiated with a full radiation dose, treatment options are limited. The majority of these patients present with surgically unresectable tumors and many experts consider these tumors incurable with less than 6 months of median survival (5). Even in surgically resected patients, the locoregional failure rate remains unsatisfactorily high with poor survival and deterioration of life quality (6). In these cases, treatment options are limited to supportive care or palliative chemotherapy with a median survival of no more than 8-10 months (7).

During the past 2 decades, the use of re-irradiation (re-RT) with or without chemotherapy for recurrent and second primary head and neck cancer has become recognized as a relatively safe and effective treatment option for highly selected patients, based on pioneering studies from the University of Chicago and Gustave-Roussy Institute $(8,9)$. Because of a lack of randomized phase III trials and a paucity of prospective multi-institutional studies, information regarding re-RT is limited and based on numerous singleinstitution reports (10-14). Many important clinical questions about the optimal use of re-RT are still unanswered. Since the introduction of helical tomotherapy at our institution in 2006, re-RT using intensity-modulated radiotherapy (IMRT) has been actively applied to patients with recurrent and second primary head and neck cancer. Herein, the clinical 
outcomes of this regimen for treatment response, patterns of failure, survival, prognostic factors, and toxicity were analyzed.

\section{Materials and Methods}

Patients. A total of 2179 consecutive patients with head and neck cancer who received RT at our institution between 2006 and 2015 were identified in our department's database. Overall, 222 patients treated with re-RT were selected. A total of 81 patients who did not receive IMRT, 48 patients who had distant metastasis at the time of re-RT, and 20 patients who received re-RT with a palliative aim ( $<40$ Gy) were excluded. The remaining 73 patients were included in our analysis.

Re-irradiation. Patients underwent simulation in the supine position and were immobilized with an aquaplastic mask device. Intravenous contrast media was administered for planning computed tomography (CT) images. RT was planned using helical tomotherapy Hi-Art System, version 2.0 (TomoTherapy Inc., Madison, WI, USA). Target volumes were as follows: planning target volume 1 (PTV1) encompassed only the gross tumor as suggested by all available images including CT, MRI, and FDG-PET; and PTV2 was defined as PTV1 plus a $5 \mathrm{~mm}$ margin. Margins were made tighter if adjacent critical organs abutted the PTV. Six patients received additional elective nodal irradiation. Median volumes were $43 \mathrm{ml}$ (range=2-540 ml) for PTV1 and $101 \mathrm{ml}$ (range=5-791 ml) for PTV2. In general, daily fraction doses were 2.0-3.0 Gy for PTV1 and 1.8-2.0 Gy for PTV2. Median total dose to PTV1 was 60 Gy (range $=40-78 \mathrm{~Gy}$ ). To compensate for effects of varying dosefractionation schedules, dose values were biologically normalized to equivalent doses in 2 Gy fractions (EQD2, a/b ratio: 10) using a linear-quadratic model. Using the image guidance system, setup errors were corrected using megavoltage CT for each RT fraction. Details of IMRT techniques in our institution are in the previous report (15).

Response and outcomes assessment. All available imaging studies including CT, MRI, ultrasound, FDG-PET, and medical records were reviewed. Tumor response after re-RT was evaluated according to the Response Evaluation Criteria in Solid Tumors (RECIST criteria). Complete or partial responses were classified as "responses". Patterns of recurrence were categorized as local, regional, or distant metastasis. Local recurrence was defined as progression of the disease at the primary site. Regional recurrence was defined as metastasis to a cervical lymph node. Distant metastasis was defined as evidence of metastasis to distant organs. We defined infield-locoregional recurrence as recurrence at the reirradiated site.

Toxicity assessment. We defined acute toxicities as events occurring within 3 months of re-RT start. Late toxicities were defined as events occurring after 3 months from re-RT start. All toxicities were graded according to the Common Terminology Criteria for Adverse Events (CTCAE, version 4.0) and toxicities rated greater than grade 3 were considered severe.

Statistical analysis. Locoregional recurrence-free survival (LRFS, time from date of first re-RT to locoregional recurrence), progression-free survival (PFS, time from date of first re-RT to any recurrence or death), and overall survival (OS, time from date of first re-RT to any cause of death) were estimated by the Kaplan-Meier method. Univariate and multivariate analyses were performed with Cox's regression model. All available variables were selected, and multivariate analysis was carried out using backwards elimination with a large alpha level (0.20) to stay within the model. To identify factors associated with severe toxicity, a Pearson chi-square test or Fisher's exact test was used. The best cut-off value of numerical variables (re-RT dose and interval between initial RT and re-RT) for survival was chosen using Youden's Index. Receiver operating characteristic (ROC) curves and the respective areas under the curves (AUC) were determined, and sensitivity, specificity, positive and negative predictive values were calculated. Statistical analyses were performed using SPSS version 23.0.0 (SPSS, Chicago, IL, USA). $p$-Values $\leq 0.05$ were considered significant.

\section{Results}

Patients. Patient characteristics for 56 men and 17 women are shown in Table I. Median age was 57 years at the time of re-RT. All tumors were confirmed histologically. Squamous cell carcinomas occurred in 51 patients $(70 \%)$. The primary tumor originated from the nasopharynx in 17 patients, maxillary sinus, nasal cavity, or paranasal sinus in 17 , oral cavity in 9 , oropharynx in 10 , hypopharynx in 5 , larynx in 9, and other sites in 6. Most patients had recurrent diseases, and 8 patients presented with a second primary tumor. Of the 73 patients, 44 had recurrence at the primary site, 15 in the neck, and 14 in both sites. No patients showed evidence of metastasis to distant organs, except a single patient with solitary resected lung metastasis. All patients had previously received full-dose irradiation. Median interval between initial RT and re-RT was 31 months (range=3-204 months). Median dose delivered during initial RT course was EQD2 66 Gy (range=42-74 Gy).

Treatment. Before re-RT, 28 (38\%) patients underwent salvage surgery (complete or partial resection), and 53 (73\%) patients received chemotherapy concurrent with re-RT, mostly with cisplatin (72\%). Ten (14\%) patients received induction chemotherapy before re-RT. The median dose delivered during the re-RT course was EQD2 $60 \mathrm{~Gy}$ (range $=48-78 \mathrm{~Gy}$ ). Median cumulative dose of the two irradiations was EQD2 126 Gy (range=90-148 Gy). Direct comparison of the initial and re-RT plans was performed to determine the overlap of the re-RT plan with prior RT treatment areas.

Response evaluation. Infield lesions were evaluated for response after re-RT completion (Table II). Of the 73 patients, 69 had available data for response evaluation. The complete remission rate was $28 \%$ and the partial remission rate was $39 \%$ (total response rate $67 \%$ ) among evaluable patients. Re-RT responses were analyzed by surgical 
Table I. Patient characteristics.

\begin{tabular}{|c|c|c|}
\hline Variables & & $\%$ \\
\hline \multicolumn{3}{|l|}{ Age (years) } \\
\hline Median, range & \multicolumn{2}{|c|}{$57(20-83)$} \\
\hline \multicolumn{3}{|l|}{ Gender } \\
\hline Male & 56 & 77 \\
\hline Female & 17 & 23 \\
\hline \multicolumn{3}{|l|}{ ECOG performance status } \\
\hline $0 \sim 1$ & 64 & 88 \\
\hline $2 \sim 3$ & 9 & 12 \\
\hline \multicolumn{3}{|l|}{ Primary site at initial diagnosis } \\
\hline Nasopharynx & 17 & 23 \\
\hline Maxillary sinus, Nasal cavity, PNS & 17 & 23 \\
\hline Oral cavity & 9 & 12 \\
\hline Oropharynx & 10 & 14 \\
\hline Hypopharynx & 5 & 7 \\
\hline Larynx & 9 & 12 \\
\hline Other & 6 & 8 \\
\hline \multicolumn{3}{|l|}{ Initial treatment } \\
\hline Surgery + PORT & 39 & 47 \\
\hline RT alone & 34 & 53 \\
\hline \multicolumn{3}{|l|}{ Progression type } \\
\hline Recurrence & 65 & 89 \\
\hline Second primary & 8 & 11 \\
\hline \multicolumn{3}{|l|}{ Progression patterns } \\
\hline Primary site & 44 & 60 \\
\hline Neck nodal site & 15 & 21 \\
\hline Both & 14 & 19 \\
\hline \multicolumn{3}{|l|}{ Months from prior RT, months } \\
\hline Median, range & \multicolumn{2}{|c|}{$31(3-204)$} \\
\hline \multicolumn{3}{|l|}{ Prior RT dose, EQD2 (Gy) } \\
\hline Median, range & \multicolumn{2}{|c|}{$66(42-74)$} \\
\hline \multicolumn{3}{|l|}{ Salvage surgery } \\
\hline Yes & 28 & 38 \\
\hline No & 45 & 62 \\
\hline \multicolumn{3}{|l|}{ Re-RT dose, EQD2 (Gy) } \\
\hline Median, range & \multicolumn{2}{|c|}{$60(48-78)$} \\
\hline \multicolumn{3}{|l|}{ Cumulative RT dose, EQD2 (Gy) } \\
\hline Median, range & \multicolumn{2}{|c|}{$126(90-148)$} \\
\hline Re-RT PTV1 volume (ml) & & \\
\hline Median, range & \multicolumn{2}{|c|}{$43(2-540)$} \\
\hline \multicolumn{3}{|l|}{ Re-RT PTV2 volume (ml) } \\
\hline Median, range & \multicolumn{2}{|c|}{$101(5-791)$} \\
\hline \multicolumn{3}{|l|}{ Induction chemotherapy } \\
\hline Yes & 10 & 14 \\
\hline \multicolumn{3}{|l|}{ Concurrent chemotherapy } \\
\hline Yes & 53 & 73 \\
\hline
\end{tabular}

ECOG: Eastern Cooperative Oncology Group; PNS: paranasal sinus; RT: radiotherapy; PORT: postoperative RT; PTV: planning target volume.

resection before re-RT. Complete response of surgically resected patients was defined as no evidence of disease status after re-RT. For 28 patients treated surgically, the complete remission rate was $42 \%$ and partial remission was $69 \%$. Overall, for non-surgical patients, the response rate was $62 \%$ and the local control rate was $95 \%$.
Table II. In-field tumor response.

\begin{tabular}{lccc}
\hline Response & $\begin{array}{c}\text { All } \\
\text { patients } \\
(\mathrm{n}=73) \\
\text { No }(\%)\end{array}$ & $\begin{array}{c}\text { Non-surgically } \\
\text { resected } \\
\text { patients }(\mathrm{n}=45) \\
\text { No }(\%)\end{array}$ & $\begin{array}{c}\text { Surgically } \\
\text { resected } \\
\text { patients }(\mathrm{n}=28) \\
\text { No }(\%)\end{array}$ \\
\hline $\mathrm{CR}$ & $19(26)$ & $8(18)$ & $11(39)^{*}$ \\
PR & $27(37)$ & $18(39)$ & $9(32)$ \\
SD & $19(26)$ & $16(36)$ & $3(11)$ \\
PD & $0(0)$ & $0(0)$ & $0(0)$ \\
UE & $8(11)$ & $3(7)$ & $5(18)$ \\
\hline
\end{tabular}

CR: Complete response; PR: partial response; SD: stable disease; PD: progressive disease; UE: un-evaluable target lesions; \%: percentage in all patients of each patient group. ${ }^{*}$ No evidence of disease (NED) status.

Table III. Failure patterns.

\begin{tabular}{lccc}
\hline Failure site & $\begin{array}{c}\text { All } \\
\text { patients } \\
(\mathrm{n}=73) \\
\text { No }(\%)\end{array}$ & $\begin{array}{c}\text { Non-surgically } \\
\text { resected } \\
\text { patients }(\mathrm{n}=45) \\
\text { No }(\%)\end{array}$ & $\begin{array}{c}\text { Surgically } \\
\text { resected } \\
\text { patients }(\mathrm{n}=28) \\
\text { No }(\%)\end{array}$ \\
\hline Total failures & $45(62)$ & $27(60)$ & $18(64)^{*}$ \\
Local only & $21(29)$ & $12(27)$ & $9(32)$ \\
Regional only & $9(12)$ & $7(16)$ & $2(7)$ \\
Local and distant only & $5(7)$ & $2(4)$ & $3(11)$ \\
Distant only & $8(11)$ & $5(11)$ & $3(11)$ \\
Distant followed by local & $2(3)$ & $1(2)$ & $1(4)$ \\
Total locoregional & $37(51)$ & $22(49)$ & $15(54)$ \\
Total infield & $25(34)$ & $15(33)$ & $10(36)$ \\
\hline
\end{tabular}

$\%$ : Percentage in all patients of each patient group. * No evidence of disease (NED) status.

Survival outcomes. At the last follow-up, 42 (58\%) patients were alive. Of the 31 patients who died, 55\% died of disease progression (13 after locoregional recurrences, 4 after systemic recurrences). The rest died of other medical conditions, sepsis after intensive cancer treatments, or unknown causes. The median follow-up was 28 months (range=6-70 months) after re-RT for surviving patients. Median OS was 33 months and the actuarial 2-year rate was $58 \%(95 \% \mathrm{CI}=46-70 \%)$. A total of 45 subsequent recurrences or disease progression after re-RT occurred during the followup period. The median PFS was 8 months and the actuarial 2year PFS rate was 20\% (95\% CI=10-30\%) (Figure 1).

Locoregional recurrence. Among 45 patients who had a third recurrence, the incidence of overall locoregional recurrence as any component of failure was $82 \%$ (37 patients), as shown in Table III. Thirty patients experienced locoregional recurrence without evidence of distant metastasis and 7 had 


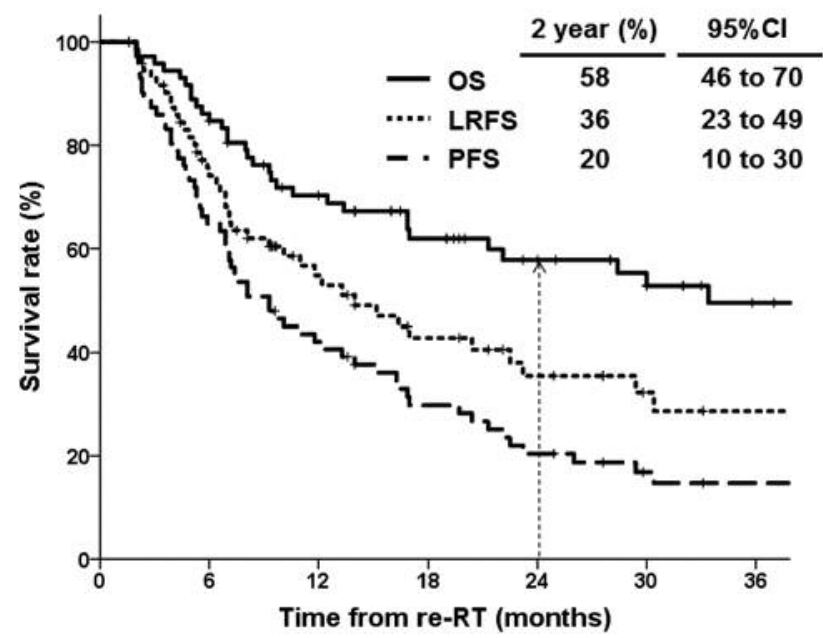

Figure 1. Overall survival (OS), locoregional recurrence-free survival (LRFS), and progression-free survival (PFS) rates for re-irradiation using helical tomotherapy of recurrent and second primary head and neck cancer $(n=73)$.

simultaneous or subsequent locoregional recurrence with distant metastasis. Twenty-five locoregional recurrences occurred within the high-dose irradiated areas and 12 were outside these areas. Patterns of failure were analyzed by surgical resection. Of the 18 patients who failed after being treated surgically, $83 \%$ experienced locoregional recurrence with $67 \%$ occurring within the high-dose irradiated areas. Similarly, in 27 patients treated non-surgically, $81 \%$ experienced locoregional recurrence with $68 \%$ occurring within the high-dose irradiated areas. Median locoregional recurrence-free survival was 23 months and the actuarial 2year LRFS rate was $36 \%(95 \% \mathrm{CI}=23-49 \%)$, as shown in Figure 1 .

Prognostic factors. The following potential prognostic variables were examined in univariate and multivariate analyses: male $v s$. female, age at the time of re-RT ( $\geq 50 v s$. $<50$ years), ECOG performance status (2-3 vs. 0-1), disease site (nasopharynx, maxillary sinus, nasal cavity, or paranasal sinus vs. other), recurrence pattern (primary, neck vs. both), surgical resection (yes vs. no), re-RT dose (EQD2, $\geq 66$ Gy vs. $<66 \mathrm{~Gy}$ ), time between first RT and re-RT ( $>2$ years $v s$. $\leq 2$ years), tumor volume ( $\geq$ median volume $v s$. $<$ median), and concurrent chemotherapy (yes $v s$. no). The most significant cut-offs for disease progressions or deaths were $66 \mathrm{~Gy}$ and 2 years for re-RT dose $(p=0.039(95 \% \mathrm{CI}=0.148-0.495))$ and time interval $(p=0.013(95 \% \mathrm{CI}=0.196-0.464))$, respectively.

In univariate analysis, re-RT dose $\geq 66 \mathrm{~Gy}$, time interval $>2$ years, and use of concurrent chemotherapy were associated with LRFS (all $p<0.05$ ). Performance status, reRT dose $\geq 66 \mathrm{~Gy}$, and time interval $>2$ years were
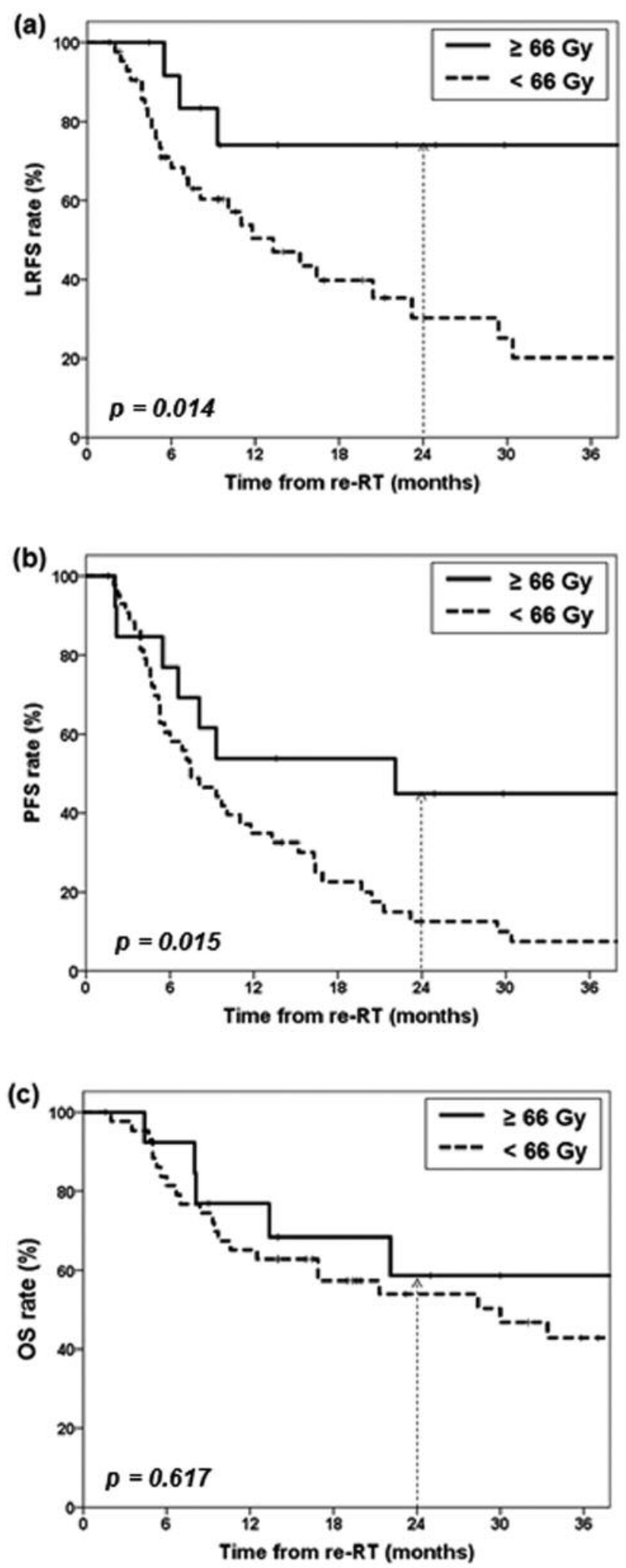

Figure 2. Locoregional recurrence-free survival (LRFS), progressionfree survival (PFS) rates, and overall survival $(O S)$ rates according to re-irradiation (re-RT) dose (EQD2<66 Gy vs. $\geq 66 \mathrm{~Gy})$. 
Table IV. Univariate and multivariate analysis.

\begin{tabular}{|c|c|c|c|c|c|c|c|c|c|c|c|c|}
\hline \multirow[t]{3}{*}{ Variable* } & \multicolumn{4}{|c|}{ LRRFS } & \multicolumn{4}{|c|}{ PFS } & \multicolumn{4}{|c|}{ OS } \\
\hline & \multicolumn{2}{|c|}{ UVA } & \multicolumn{2}{|c|}{ MVA } & \multicolumn{2}{|c|}{ UVA } & \multicolumn{2}{|c|}{ MVA } & \multicolumn{2}{|c|}{ UVA } & \multicolumn{2}{|c|}{ MVA } \\
\hline & $\begin{array}{c}\mathrm{HR} \\
(95 \% \mathrm{CI})\end{array}$ & $p$-Value & $\begin{array}{c}\mathrm{HR} \\
(95 \% \mathrm{CI})\end{array}$ & $p$-Value & $\begin{array}{c}\mathrm{HR} \\
(95 \% \mathrm{CI})\end{array}$ & $p$-Value & $\underset{(95 \% \mathrm{CI})}{\mathrm{HR}}$ & $p$-Value & $\begin{array}{c}\mathrm{HR} \\
(95 \% \mathrm{CI})\end{array}$ & $p$-Value & $\underset{(95 \% \mathrm{CI})}{\mathrm{HR}}$ & $p$-Value \\
\hline Gender & $\begin{array}{c}0.717 \\
(0.351-1.466)\end{array}$ & 0.362 & & & $\begin{array}{c}0.809 \\
(0.436-1.499)\end{array}$ & 0.500 & & & $\begin{array}{c}0.921 \\
(0.397-2.141)\end{array}$ & 0.849 & & \\
\hline Age $\geq 50$ years & $\begin{array}{c}0.642 \\
(0.317-1.298)\end{array}$ & 0.217 & & & $\begin{array}{c}0.625 \\
(0.348-1.121)\end{array}$ & 0.115 & & & $\begin{array}{c}1.308 \\
(0.535-3.195)\end{array}$ & 0.556 & & \\
\hline ECOG PS 2-3 & $\begin{array}{c}1.668 \\
(0.502-5.539)\end{array}$ & 0.403 & & & $\begin{array}{c}3.179 \\
(1.466-6.895)\end{array}$ & 0.003 & $\begin{array}{c}3.140 \\
(1.411-6.986)\end{array}$ & 0.005 & $\begin{array}{c}4.180 \\
(1.657-10.541)\end{array}$ & 0.002 & $\begin{array}{c}3.768 \\
(1.461-9.723)\end{array}$ & 0.006 \\
\hline Disease site & $\begin{array}{c}1.032 \\
(0.888-1.200)\end{array}$ & 0.679 & & & $\begin{array}{c}1.043 \\
(0.921-1.181)\end{array}$ & 0.509 & & & $\begin{array}{c}0.972 \\
(0.818-1.154)\end{array}$ & 0.745 & & \\
\hline $\begin{array}{l}\text { Recurrence } \\
\text { pattern }\end{array}$ & $\begin{array}{c}1.005 \\
(0.673-1.501)\end{array}$ & 0.980 & & & $\begin{array}{c}1.014 \\
(0.723-1.420)\end{array}$ & 0.937 & & & $\begin{array}{c}1.287 \\
(0.834-1.986)\end{array}$ & 0.254 & & \\
\hline Surgery & $\begin{array}{c}0.929 \\
(0.497-1.737)\end{array}$ & 0.818 & & & $\begin{array}{c}0.730 \\
(0.429-1.242)\end{array}$ & 0.246 & & & $\begin{array}{c}0.326 \\
(0.140-0.759)\end{array}$ & 0.009 & $\begin{array}{c}0.362 \\
(0.153-0.857)\end{array}$ & 7) \\
\hline $\begin{array}{l}\text { re-RT dose } \\
66 \mathrm{~Gy}\end{array}$ & $\begin{array}{c}0.275 \\
(0.098-0.773)\end{array}$ & 0.014 & $\begin{array}{c}0.341 \\
.120-0.96\end{array}$ & 67) & $\begin{array}{c}0.393 \\
(0.186-0.833)\end{array}$ & 0.015 & $\begin{array}{c}0.502 \\
(0.233-1.081)\end{array}$ & 0.078 & $\begin{array}{c}0.797 \\
(0.327-1.943)\end{array}$ & 0.617 & & \\
\hline $\begin{array}{l}\text { Time interval } \\
>2 \text { years }\end{array}$ & $\begin{array}{c}0.372 \\
(0.199-0.697)\end{array}$ & 0.002 & $\begin{array}{c}0.396 \\
.209-0.75\end{array}$ & 50) & $\begin{array}{c}0.439 \\
(0.261-0.739)\end{array}$ & 0.002 & $\begin{array}{c}0.452 \\
(0.266-0.768)\end{array}$ & 0.003 & $\begin{array}{c}0.351 \\
(0.168-0.735)\end{array}$ & 0.006 & $\begin{array}{c}0.341 \\
(0.163-0.715)\end{array}$ & 0.004 \\
\hline $\begin{array}{l}\text { Tumor volume } \\
\text { median } \geq 43 \mathrm{cc}\end{array}$ & $\begin{array}{c}0.916 \\
(0.494-1.696)\end{array}$ & 0.779 & & & $\begin{array}{c}1.083 \\
(0.649-1.806)\end{array}$ & 0.760 & & & $\begin{array}{c}1.758 \\
(0.856-3.611)\end{array}$ & 0.124 & & \\
\hline Concurrent CTx & $\begin{array}{c}0.498 \\
(0.263-0.943)\end{array}$ & $\begin{array}{l}0.032 \\
(0\end{array}$ & $\begin{array}{c}0.461 \\
.242-0.87\end{array}$ & 78) & $\begin{array}{c}0.695 \\
(0.399-1.212)\end{array}$ & 0.200 & & & $\begin{array}{c}1.044 \\
(0.480-2.273)\end{array}$ & 0.913 & & \\
\hline
\end{tabular}

LRFS: Loco-regional recurrence-free survival; PFS: progression-free survival; OS: overall survival; HR: hazards ratio; CI: confidence interval; RT: radiotherapy; CTx: chemotherapy. *Variables included gender (male $v s$. female), age at the time of re-RT ( $\geq 50 v s$. $<50$ years), ECOG performance status (2-3 vs. 0-1), disease site (nasopharynx/ maxillary sinus/ nasal cavity/ paranasal sinus $v s$. others), Recurrence pattern (primary $v s$. neck $v s$. both), surgical resection (yes $v s$. no), re-RT dose ( $\geq \mathrm{EQD} 266 \mathrm{~Gy} v s .<\mathrm{EQD} 266 \mathrm{~Gy}$ ), the time interval between first RT and re-RT ( $>2$ years $v s . \leq 2$ years), tumor volume ( $\geq$ median volume $v s .<$ median), and concurrent chemotherapy (yes $v s$. no).

associated with PFS (all $p<0.05$ ). Performance status, use of surgical resection, and interval $>2$ years were associated with OS (all $p<0.05)$. In multivariate analysis, re-RT dose $\geq 66$ Gy, time interval $>2$ years, and use of concurrent chemotherapy were associated with improved LRFS. Performance status and time interval $>2$ years were remained independent prognostic factors in PFS. Additionally, use of surgical resection was also associated with better OS (Table IV).

There were 16 patients who received higher dose $(\geq 66$ Gy) and 57 patients received lower dose ( $<66 \mathrm{~Gy})$. When the time interval was longer than 2 years, the patients tended to be prescribed higher doses $(11 / 38,29 \%$ vs. 5/35, $14 \%$ $p=0.130)$. The 2-year LRFS rates were $73 \%$ and $26 \%$ and 2 year PFS rates were $48 \%$ and $13 \%$ in patients with $\geq 66$ Gy and $<66$ Gy, respectively ( $p=0.014$ and 0.015 , respectively) (Figure 2). The overall recurrence rates were significantly lower with higher doses $(31 \%$ vs. $70 \%, p=0.005)$. The locoregional recurrence rates among them were also significantly lower $(19 \%$ vs. $60 \%, p=0.004)$. Although it was not significantly different, the number of infield failures among locoregional recurrences was smaller with higher doses (2/3 vs. 23/34) (Figure 3). The 2-year OS rates were not significantly different $(59 \%$ and $58 \%, p=0.617$, Figure $2)$. However, there were only 2 patients who died of locoregional progression among 6 patients who died after receiving $\geq 66 \mathrm{~Gy}$. If locoregional control was done with sufficient dose of radiation and sufficient re-RT interval, overall survival may have been improved.

Toxicity. Of 70 evaluable patients, 10 (14\%) had clinically significant acute toxicity during re-RT. Two patients had a combination of grade 3 dyspnea and grade 3 aspiration or laryngeal edema leading to hospitalization for tracheostomy. One patient developed grade 3 oral pain and grade 2 edema face, and another had grade 3 dyspnea with grade 2 neck edema. The rest 6 patients had grade 3 skin toxicity or mucositis. Poor performance status was the significant determinant of clinically significant acute toxicity $(p=0.017)$ and recurrent tumors at oropharynx were the most common $(50 \%)$ among them. Although it was not statistically different, the volume of gross tumor was larger in patients 


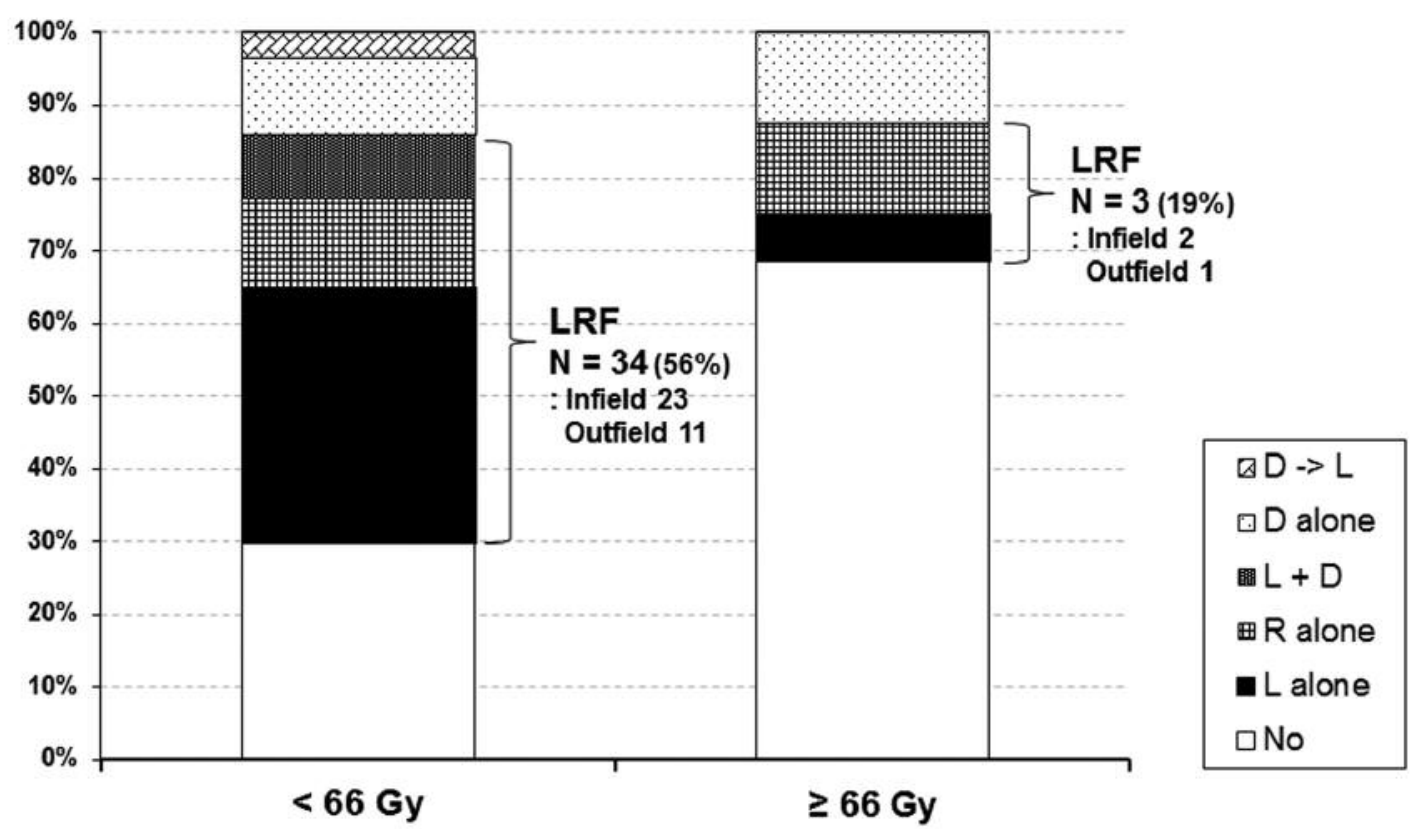

Figure 3. Pattern of failure according to re-irradiation (re-RT) dose (EQD2<66 Gy vs. $\geq 66$ Gy). LRF: Locoregional failure; D: distant failure; L: local failure; $R$ : regional failure.

Table V. Late severe complications after re-irradiation using helical tomotherapy.

\begin{tabular}{|c|c|c|c|c|c|c|c|c|c|c|}
\hline Patient & Diagnosis & $\begin{array}{l}\text { Treatment } \\
\text { site }\end{array}$ & $\begin{array}{c}\text { Initial } \\
\text { treatment }\end{array}$ & $\begin{array}{l}\text { Salvage } \\
\text { treatment }\end{array}$ & $\begin{array}{c}\text { re-RT } \\
\text { dose } \\
(\mathrm{EQD} 2)\end{array}$ & $\begin{array}{c}\text { Cumulative } \\
\text { dose } \\
\text { (EQD2) }\end{array}$ & $\begin{array}{l}\text { GTV } \\
\text { volume } \\
(\mathrm{ml})\end{array}$ & ENI & $\begin{array}{l}\text { Interval from } \\
\text { previous RT } \\
\text { (months) }\end{array}$ & Complication \\
\hline $\mathrm{M} / 45$ & NPC & Primary & RT & CCRT & 52 & 122 & 53 & No & 32 & Middle ear inflammation G3 \\
\hline $\mathrm{M} / 66$ & NPC & Both & RT & $\mathrm{IC}+\mathrm{CRT}$ & 66 & 140 & 35 & Yes & 204 & Trismus G3 \\
\hline $\mathrm{M} / 59$ & MSC & Primary & RT & $\mathrm{Op}+\mathrm{CRT}$ & 50 & 118 & 89 & No & 62 & Hearing impairment G3 \\
\hline $\mathrm{M} / 83$ & MSC & Primary & $\mathrm{RT}+\mathrm{Op}$ & RT alone & 74 & 140 & 48 & No & 8 & Trismus G3, Dry eye G3 \\
\hline $\mathrm{M} / 46$ & $\mathrm{OC}$ & Both & $\mathrm{Op}+\mathrm{RT}$ & CCRT & 64 & 128 & 540 & No & 3 & Edema face G3, Facial pain G3 \\
\hline $\mathrm{F} / 51$ & OC & Neck & $\mathrm{Op}+\mathrm{RT}$ & CCRT & 64 & 128 & 64 & No & 3 & Neck pain G3, Neck soft tissue necrosis G3 \\
\hline $\mathrm{M} / 55$ & OPC & Both & RT & $\mathrm{IC}+\mathrm{CRT}$ & 64 & 134 & 60 & No & 24 & Dysphagia G3, Skin ulceration G3 \\
\hline $\mathrm{M} / 74$ & $\mathrm{LC}$ & Neck & RT & CCRT & 62 & 132 & 13 & No & 70 & Dysphagia G4, Oral pain G3 \\
\hline $\mathrm{M} / 83$ & LC & Primary & RT & CCRT & 68 & 134 & 52 & Yes & 93 & Dyspnea G4 \\
\hline $\mathrm{F} / 48$ & SDC & Primary & $\mathrm{Op}+\mathrm{RT}$ & CCRT & 60 & 114 & 30 & No & 63 & Dysphagia G3 \\
\hline $\mathrm{M} / 50$ & OPC & Primary & $\mathrm{Op}+\mathrm{RT}$ & CCRT & 60 & 127 & 31 & No & 113 & Dysphagia G3 \\
\hline $\mathrm{F} / 39$ & NPC & Both & RT & CCRT & 45 & 115 & 19 & No & 31 & Hearing impairment G3 \\
\hline $\mathrm{M} / 59$ & NPC & Both & RT & CCRT & 67 & 138 & 12 & No & 14 & Neck soft tissue necrosis G3 \\
\hline $\mathrm{M} / 50$ & $\mathrm{OC}$ & Neck & $\mathrm{Op}+\mathrm{RT}$ & $\mathrm{Op}+\mathrm{CRT}$ & 60 & 124 & 42 & No & 13 & Xerostomia G3 \\
\hline
\end{tabular}

NPC: Nasopharynx cancer; MSC: maxillary sinus cancer; OC: oral cavity cancer; OPC: oropharynx cancer; LC: larynx cancer; SDC: salivary ductal cancer; CCRT: concurrent chemo-radiotherapy; IC: induction chemotherapy; CRT: chemo-radiotherapy; EQD2: equivalent doses in 2 Gy fractions; GTV: gross tumor volume; ENI: elective nodal irradiation.

who experienced clinically significant acute toxicities (mean $51 \mathrm{ml} v s .128 \mathrm{ml}, p=0.167)$. Of 63 evaluable patients, 14 (22\%) showed clinically significant late toxicity (Table V). No grade 5 toxicity was observed and no association with severe late toxicity was identified.

\section{Discussion}

This study explored the treatment outcomes of recurrent and second primary head and neck cancer patients whose gross tumor volume was re-irradiated with small margins using 
IMRT and daily image guidance. Our study showed 2-year OS and LRFS rates of $58 \%$ and $36 \%$ respectively, with a median survival of 33 months. Severe late complications occurred in $22 \%$ of evaluable patients, but most toxicities were manageable with no treatment-related mortalities. Additionally, several important clinical parameters were identified as prognostic factors in our cohort. Higher radiation dose, sufficient time from first RT to re-RT, and use of chemotherapy concurrent with re-RT were significantly associated with better locoregional control. In addition to these factors, good performance status, and additional surgical resection were significantly related to improved survival.

The primary concerns of physicians regarding the decision to use re-RT have been toxicity and safety. In the French GETTEC study, $39 \%$ of patients who underwent surgery plus re-RT and $10 \%$ in the surgery alone arm experienced grade 3 or 4 late toxicity $(p=0.06)(16)$. The main toxicities included osteoradionecrosis, trismus, and sclerosis. In the Radiation Therapy Oncology Group (RTOG) 9610 multiinstitutional study, late toxicity was grade 3 in $19 \%$ of patients and grade 4 in 3\% (17). The majority of cases were associated with re-RT of the pharynx/esophagus. In the RTOG 9911 study, 5\% experienced early grade 5 toxicities and with late toxicities of grade 3 in $17 \%$, grade 4 in $17 \%$, and grade 5 in $4 \%$ (18). This study estimated that $85 \%$ of patients experienced grade 3 to 5 toxicity within the first 2 years after re-RT. In other retrospective studies, grade 3 and 4 late toxic effects ranged from $18 \%$ to $38 \%(10-13,19)$. Rupture of the carotid artery is a major concern with re-RT; McDonald et al. found an incidence of approximately $3 \%$ and most occurrences were fatal (20).

Severe toxicity could be mainly attributed to re-RT volume. In GETTECT, RTOG trials and other trials, gross disease was generally encompassed with a minimal margin of $2.0 \mathrm{~cm}$. Limiting re-RT volume to recurrent gross tumor volume with tight margins could help decrease late toxicity. A Michigan study found that all locoregional recurrences occurred within the re-irradiated infield area, and verified that confining re-RT targets to the gross tumor did not compromise tumor control (21). With the availability of more advanced RT techniques, the use of intensity modulated radiotherapy (IMRT) has been effective in sparing normal structures from excessive radiation exposure compared to conventional RT techniques $(14,22)$. An MD Anderson report on the outcomes of re-RT using IMRT (11) found that even though 1-2 cm circumferential margins were used, morbidity was significant, although possibly less severe than conventional RT. In more recent studies using both modern RT and tight margin (23-25), the 1-year local control and OS rates were $71-75 \%$ and $50-67 \%$, respectively, with mild toxicity except one paper (24). There was $32 \%$ of grade 3 or worse toxicities including six grade 5 toxicities, that required further investigation.
In our cohort, the incidence of toxicity after re-RT was in the range reported in other studies, although direct comparisons are impossible. We believe that our use of IMRT with tight margins might have contributed to the absence of treatment-related mortality. Several potential risk factors were examined and performance status and gross tumor volume were identified as clinically relevant predictive factors for acute toxicity. These findings suggest caution when treating large tumors or tumors in a complicate location. However, similar to other reports, identification of clinically relevant factors for late toxicity after re-RT remains elusive.

In the GETTEC trial, $38 \%$ of patients in the arm receiving surgery plus re-RT developed locoregional recurrences, and the 1-year loco-regional control rate was approximately $60 \%$ (16). In the RTOG 9911 trial, $31 \%$ of patients who received twice-daily re-RT without surgical resection (60 Gy in 1.5 Gy per fraction bid) experienced locoregional recurrences (7). In an MD Anderson study which included $27 \%$ of surgically resected patients, $35 \%$ of the re-RT patients had locoregional recurrences with a $64 \%$ probability of locoregional control for 2 years (12). Our study showed a locoregional recurrence rate of $51 \%$ with a 1-, 2-year LRFS rates of 55\%,36\%, respectively, similar to previous studies using re-RT. Among 37 locoregional recurrences, $32 \%$ of patients had an outfield recurrence that remained outfield if the target was treated with a $2 \mathrm{~cm}$ margin. Despite our relatively small sample size, we observed a similar distribution of failure patterns according to surgical resection. These results support the utility of tight margins.

A study examining multiple covariates in univariate and multivariate analyses found that only nonmodifiable clinical factors of male gender, and age at the time of re-RT were predictors of outcome (12). In the Gustave-Roussy Institute study, they found that irradiated volume $\left(\geq 650 \mathrm{~cm}^{3}\right)$ was the only factor associated with risk of death (relative risk, 1.8) (4). In our study, surgical resection before re-RT which could reduce the irradiation volume, and performance status were correlated with OS on multivariate analysis. Similarly, recent papers $(26,27)$ also have demonstrated that significant medical comorbidities or poor performance status could influence survival, which means that these are worse candidates for re-RT.

Modifiable prognostic factors in our study were re-RT dose, time between the two RT courses, and use of concurrent chemotherapy. Prediction of outcome remained significant when the analysis was performed with time as a continuous variable and longer time intervals were associated with better prognosis. One French review paper (28) identified that surgical resection, a dose higher than 50 to $60 \mathrm{~Gy}$ in a smaller-irradiated volume, and an interval between the two treatments of more than 2 years, were the most commonly 
highlighted prognostic factors. Two recent papers $(27,29)$ also showed that the patients receiving more than $40 \mathrm{~Gy}$ in 5 fractions (BED equal to $72 \mathrm{~Gy}$ ) had significantly higher local control than others. Although more investigation is needed on the optimal dose and fractionation schedule, our study is worth noting in this context.

In interpreting our data, limitations of retrospective analyses and patient and treatment selection must be considered. The reasons that our patients were treated with re-RT using helical tomotherapy were unclear, but the use of this regimen might be reliably recommended to patients who have factors suggesting better outcome. Our cohort was heterogeneous, so the results cannot be directly applied to all recurrent head and neck cancers to encourage the widespread use of re-RT. Since the cohort was relatively small, conducting a complete statistical analysis was limited. The prognostic factors identified in our data must be verified. Nevertheless, the strength of our study is that our patients received relatively homogenous treatment including re-RT with tight margins and sufficient radiation dose, performed at a single institution and were followedup for a long period of time.

\section{Conclusion}

Moderate safety and acceptable acute and late toxicity after re-RT using full radiation dose, tight margins, and daily image guidance is reported. Encouraging local control and survival were obtained similar to historical data using these modern RT strategies. Resources should be directed toward prospective studies of re-RT in this field.

\section{Conflicts of Interest}

The Authors declare that they have no competing interests regarding this study.

\section{Acknowledgements}

None.

\section{References}

1 Bernier J, Domenge C, Ozsahin M, Matuszewska K, Lefebvre JL, Greiner RH, Giralt J, Maingon P, Rolland F, Bolla M, Cognetti F, Bourhis J, Kirkpatrick A and van Glabbeke M: Postoperative irradiation with or without concomitant chemotherapy for locally advanced head and neck cancer. $\mathrm{N}$ Engl J Med 350(19): 1945-1952, 2004.

2 Cooper JS, Pajak TF, Forastiere AA, Jacobs J, Campbell BH, Saxman SB, Kish JA, Kim HE, Cmelak AJ, Rotman M, Machtay M, Ensley JF, Chao KS, Schultz CJ, Lee $\mathrm{N}$ and Fu KK: Postoperative concurrent radiotherapy and chemotherapy for high-risk squamous-cell carcinoma of the head and neck. N Engl J Med 350(19): 1937-1944, 2004.
3 Setton J, Caria N, Romanyshyn J, Koutcher L, Wolden SL, Zelefsky MJ, Rowan N, Sherman EJ, Fury MG, Pfister DG, Wong RJ, Shah JP, Kraus DH, Shi W, Zhang Z, Schupak KD, Gelblum DY, Rao SD and Lee NY: Intensity-modulated radiotherapy in the treatment of oropharyngeal cancer: an update of the Memorial Sloan-Kettering Cancer Center experience. Int J Radiat Oncol Biol Phys 82(1): 291-298, 2012.

4 Garden AS, Dong L, Morrison WH, Stugis EM, Glisson BS, Frank SJ, Beadle BM, Gunn GB, Schwartz DL, Kies MS, Weber RS, Ang KK and Rosenthal DI: Patterns of disease recurrence following treatment of oropharyngeal cancer with intensity modulated radiation therapy. Int J Radiat Oncol Biol Phys 85(4): 941-947, 2013.

5 Taussky D, Dulguerov P and Allal AS: Salvage surgery after radical accelerated radiotherapy with concomitant boost technique for head and neck carcinomas. Head Neck 27(3): 182-186, 2005.

6 Temam S, Koka V, Mamelle G, Julieron M, Carmantrant R, Marandas P, Janot F, Bourhis J and Luboinski B: Treatment of the N0 neck during salvage surgery after radiotherapy of head and neck squamous cell carcinoma. Head Neck 27(8): 653-658, 2005.

7 Vermorken JB, Mesia R, Rivera F, Remenar E, Kawecki A, Rottey S, Erfan J, Zabolotnyy D, Kienzer HR, Cupissol D, Peyrade F, Benasso M, Vynnychenko I, De Raucourt D, Bokemeyer C, Schueler A, Amellal N and Hitt R: Platinumbased chemotherapy plus cetuximab in head and neck cancer. $\mathrm{N}$ Engl J Med 359(11): 1116-1127, 2008.

8 De Crevoisier R, Bourhis J, Domenge C, Wibault P, Koscielny S, Lusinchi A, Mamelle G, Janot F, Julieron M, Leridant AM, Marandas P, Armand JP, Schwaab G, Luboinski B and Eschwege F: Full-dose reirradiation for unresectable head and neck carcinoma: experience at the Gustave-Roussy Institute in a series of 169 patients. J Clin Oncol 16(11): 3556-3562, 1998.

9 Brockstein B, Haraf DJ, Stenson K, Fasanmade A, Stupp R, Glisson B, Lippman SM, Ratain MJ, Sulzen L, Klepsch A, Weichselbaum RR and Vokes EE: Phase I study of concomitant chemoradiotherapy with paclitaxel, fluorouracil, and hydroxyurea with granulocyte colony-stimulating factor support for patients with poor-prognosis cancer of the head and neck. $J$ Clin Oncol 16(2): 735-744, 1998.

10 Salama JK, Vokes EE, Chmura SJ, Milano MT, Kao J, Stenson KM, Witt ME and Haraf DJ: Long-term outcome of concurrent chemotherapy and reirradiation for recurrent and second primary head-and-neck squamous cell carcinoma. Int J Radiat Oncol Biol Phys 64(2): 382-391, 2006.

11 Sulman EP, Schwartz DL, Le TT, Ang KK, Morrison WH, Rosenthal DI, Ahamad A, Kies M, Glisson B, Weber R and Garden AS: IMRT reirradiation of head and neck cancer-disease control and morbidity outcomes. Int J Radiat Oncol Biol Phys 73(2): 399-409, 2009.

12 Roh KW, Jang JS, Kim MS, Sun DI, Kim BS, Jung SL, Kang JH, Yoo EJ, Yoon SC, Jang HS, Chung SM and Kim YS: Fractionated stereotactic radiotherapy as reirradiation for locally recurrent head and neck cancer. Int J Radiat Oncol Biol Phys 74(5): 1348-1355, 2009.

13 Rusthoven KE, Feigenberg SJ, Raben D, Kane M, Song JI, Nicolaou N, Mehra R, Burtness B, Ridge J, Swing R, Lango M, Cohen R, Jimeno A and Chen C: Initial results of a Phase I doseescalation trial of concurrent and maintenance erlotinib and reirradiation for recurrent and new primary head-and-neck cancer. Int J Radiat Oncol Biol Phys 78(4): 1020-1025, 2010. 
14 Leong YH, Soon YY, Lee KM, Wong LC, Tham IWK and Ho FCH: Long-term outcomes after reirradiation in nasopharyngeal carcinoma with intensity-modulated radiotherapy: A metaanalysis. Head Neck 4O(3): 622-631, 2017.

15 Lee IJ, Seong J, Lee CG, Kim YB, Keum KC, Suh CO, Kim GE and Cho J: Early clinical experience and outcome of helical tomotherapy for multiple metastatic lesions. Int J Radiat Oncol Biol Phys 73(5): 1517-1524, 2009.

16 Janot F, de Raucourt D, Benhamou E, Ferron C, Dolivet G, Bensadoun RJ, Hamoir M, Gery B, Julieron M, Castaing M, Bardet E, Gregoire V and Bourhis $\mathrm{J}$ : Randomized trial of postoperative reirradiation combined with chemotherapy after salvage surgery compared with salvage surgery alone in head and neck carcinoma. J Clin Oncol 26(34): 5518-5523, 2008.

17 Spencer SA, Harris J, Wheeler RH, Machtay M, Schultz C, Spanos W, Rotman M, Meredith R and Ang KK: Final report of RTOG 9610, a multi-institutional trial of reirradiation and chemotherapy for unresectable recurrent squamous cell carcinoma of the head and neck. Head Neck 30(3): 281-288, 2008.

18 Langer CJ, Harris J, Horwitz EM, Nicolaou N, Kies M, Curran W, Wong S and Ang K: Phase II study of low-dose paclitaxel and cisplatin in combination with split-course concomitant twice-daily reirradiation in recurrent squamous cell carcinoma of the head and neck: results of Radiation Therapy Oncology Group Protocol 9911. J Clin Oncol 25(30): 4800-4805, 2007.

19 Lee N, Chan K, Bekelman JE, Zhung J, Mechalakos J, Narayana A, Wolden S, Venkatraman ES, Pfister D, Kraus D, Shah J and Zelefsky MJ: Salvage re-irradiation for recurrent head and neck cancer. Int J Radiat Oncol Biol Phys 68(3): 731-740, 2007.

20 McDonald MW, Moore MG and Johnstone PA: Risk of carotid blowout after reirradiation of the head and neck: a systematic review. Int J Radiat Oncol Biol Phys 82(3): 1083-1089, 2012.

21 Popovtzer A, Gluck I, Chepeha DB, Teknos TN, Moyer JS, Prince ME, Bradford CR and Eisbruch A: The pattern of failure after reirradiation of recurrent squamous cell head and neck cancer: implications for defining the targets. Int J Radiat Oncol Biol Phys 74(5): 1342-1347, 2009.

22 Vargo JA, Ward MC, Caudell JJ, Riaz N, Dunlap NE, Isrow D, Zakem SJ, Dault J, Awan MJ, Higgins KA, Hassanadeh C, Beitler JJ, Reddy CA, Marcrom S, Boggs DH, Bonner JA, Yao M, Machtay M, Siddiqui F, Trotti AM, Lee NY, Koyfman SA, Ferris RL and Heron DE: A multi-institutional comparison of SBRT and IMRT for definitive reirradiation of recurrent or second primary head and neck cancer. Int J Radiat Oncol Biol Phys 100: 595-605, 2017.
23 Rades D, Bartscht T, Idel C and Hakim SG: Re-irradiation with 36 Gy (1.5 Gy Twice Daily) plus paclitaxel for advanced recurrent and previously irradiated SCCHN is feasible. Anticancer Res 38(1): 519-523, 2018.

24 Yamazaki H, Demizu Y, Okimoto T, Ogita M, Himei K, Nakamura S, Suzuki G, Yoshida K, Kotsuma T and Yoshioka Y: Comparison of re-irradiation outcomes for charged particle radiotherapy and robotic stereotactic radiotherapy using cyberknife for recurrent head and neck cancers: A Multi-institutional matched-cohort analysis. Anticancer Res 36(10): 5507-5514, 2016.

25 Rades D, Seidl D, Wollenberg B, Schild SE and Hakim SG: Radiochemotherapy with paclitaxel for recurrent previously irradiated squamous cell carcinoma of the head and neck. Anticancer Res 36(10): 5463-5468, 2016.

26 Tanvetyanon T, Padhya T, McCaffrey J, Zhu W, Boulware D, Deconti R and Trotti A: Prognostic factors for survival after salvage reirradiation of head and neck cancer. J Clin Oncol 27(12): 1983-1991, 2009.

27 Buglione M, Maddalo M, Mazzeo E, Bonomo P, Spiazzi L, Bruni A, Paiar F, Triggiani L, Greto D, Rubino L, Livi L, Bertoni $F$ and Magrini SM: Reirradiation in head and neck recurrent or second primary tumor: efficacy, safety, and prognostic factors. Tumori 101(5): 585-592, 2015.

28 Ohnleiter T, Truntzer P, Antoni D, Guihard S, Elgard AM and Noel G: Prognostic factors for head and neck cancer reirradiation: A systematic review. Cancer Radiother 21(4): 316338, 2017.

29 Rwigema JC, Heron DE, Ferris RL, Andrade RS, Gibson MK, Yang Y, Ozhasoglu C, Argiris AE, Grandis JR and Burton SA: The impact of tumor volume and radiotherapy dose on outcome in previously irradiated recurrent squamous cell carcinoma of the head and neck treated with stereotactic body radiation therapy. Am J Clin Oncol 34(4): 372-379, 2011.
Received March 3, 2018

Revised March 22, 2018

Accepted March 23, 2018 